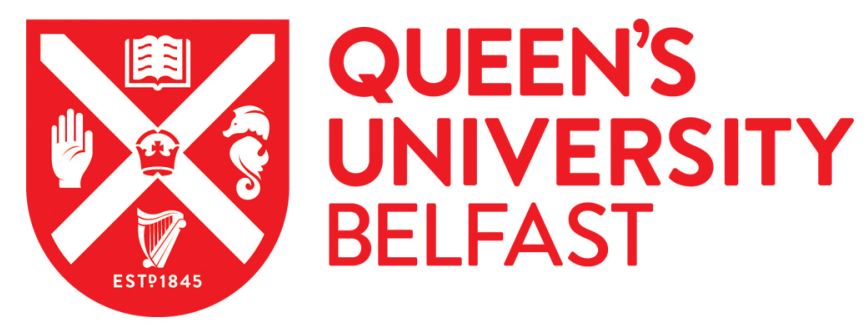

\title{
Preparation and characterisation of poly(ethylene glycol)-adsorbed graphene oxide nanosheets
}

Al-Bermany, E., \& Chen, B. (2021). Preparation and characterisation of poly(ethylene glycol)-adsorbed graphene oxide nanosheets. Polymer International, 70(3), 341-351. https://doi.org/10.1002/pi.6140

Published in:

Polymer International

Document Version:

Peer reviewed version

Queen's University Belfast - Research Portal:

Link to publication record in Queen's University Belfast Research Portal

Publisher rights

(C) 2020 John Wiley \& Sons.

This work is made available online in accordance with the publisher's policies. Please refer to any applicable terms of use of the publisher.

\section{General rights}

Copyright for the publications made accessible via the Queen's University Belfast Research Portal is retained by the author(s) and / or other copyright owners and it is a condition of accessing these publications that users recognise and abide by the legal requirements associated with these rights.

Take down policy

The Research Portal is Queen's institutional repository that provides access to Queen's research output. Every effort has been made to ensure that content in the Research Portal does not infringe any person's rights, or applicable UK laws. If you discover content in the Research Portal that you believe breaches copyright or violates any law, please contact openaccess@qub.ac.uk. 


\title{
Preparation and Characterization of Poly(ethylene glycol)-Adsorbed Graphene Oxide Nanosheets
}

\author{
Ehssan Al-Bermany ${ }^{1,3}$, Biqiong Chen ${ }^{2 *}$ \\ ${ }^{1}$ Department of Materials Science and Engineering, the University of Sheffield, Mappin Street, \\ Sheffield S1 3JD, United Kingdom. \\ ${ }^{2}$ School of Mechanical and Aerospace Engineering, Queen's University Belfast, Stranmillis Road, \\ Belfast BT9 5AH, United Kingdom. \\ ${ }^{3}$ Department of Physics, College of Education for pure sciences, University of Babylon, Babylon, Iraq. \\ *Corresponding Author. Email Address: b.chen@qub.ac.uk
}

\begin{abstract}
Polymer-graphene nanocomposites are attracting growing attention of scientists and engineers as graphene-based nanofillers may enhance the properties of polymer significantly. This study aims to understand the adsorption behaviour of polymers on graphene oxide (GO) nanosheets. GO is synthesized using Hummer's method by oxidizing graphite. Poly(ethylene glycol)s (PEGs) with different molecular weights are used as polymer models. A series of PEG/GO nanohybrids are prepared by applying different parameters in the solution processing method. Fourier transform infrared spectroscopy, X-ray diffraction, differential scanning calorimetry, thermogravimetric analysis, polarized optical microscopy, scanning electron microscopy and atomic force microscopy are used for characterizing the hybrid nanomaterials. The characterization results confirm the successful preparation of $\mathrm{GO}$ and the adsorption of the PEGs onto GO. The maximum amount of the adsorbed PEG was $38 \mathrm{wt} \%$. The adsorption amount of PEG increases by $46 \%$ after reducing the mixing time from $192 \mathrm{~h}$ to $72 \mathrm{~h}, 1700 \%$ due to an increase in the molecular weight from $1 \mathrm{k}$ to $100 \mathrm{k}, 13 \%$ for doubling the mixing ratio of PEG:GO from 1.5:1 to 3:1, 44\% for applying no further washing procedure and $73 \%$ for applying all these parameters concurrently. The adsorption onto GO reduces the crystallinity of PEGs due to chain confinement. Different surface morphologies are observed in the hybrid nanomaterials showing various thicknesses of the PEG layer adsorbed on the GO nanosheets. This study may offer new insights into the manipulation of the interface in polymer-GO nanocomposites.
\end{abstract} KEYWORDS: graphene; poly(ethylene glycol); nanocomposite; surface adsorption; interface

\section{INTRODUCTION}

Polymer nanocomposites have triggered an increasing interest among scientists and engineers over the last two decades ${ }^{1,2}$. They contain a filler of nanometre size which exhibits unique advantages with regards to the enhancements of the polymer properties, such as mechanical ${ }^{3}$, thermal, electrical and/or magnetic properties, without losing optical transparency ${ }^{4}$, when compared to their polymer counterparts ${ }^{2,5}$. The properties of a polymer nanocomposite not only depend on the structure and properties of its constituents but also their interfacial characteristics ${ }^{6,7}$.

Recently, significant attention has been given to carbon-based nanomaterials; in particular, carbon nanotubes and graphene $(G)$ nanosheets as reinforcing fillers for polymers ${ }^{1}$. Graphene single nanosheet consists of honeycomb lattices with an elastic modulus of $1 \mathrm{TPa}$, the fracture strength of about $130 \mathrm{GPa}$ and thermal conductivity up to $5000 \mathrm{~W} \mathrm{mK}^{-18}$. Graphene oxide (GO) has oxygenated 
functional groups, such as epoxy and hydroxyl groups on the basal plane and carboxylic acid groups along sheet edges ${ }^{9}$. These functional groups, together with the large surface area to volume ratio ${ }^{10}$ and other excellent physical properties ${ }^{11}$, render GO nanosheets as attractive fillers ${ }^{12}$.

The polymer at the interface constitutes a significant volume fraction of the nanocomposites ${ }^{4}$. Therefore, understanding the interface and the polymer near the interface is considered of paramount importance ${ }^{11}$. The degree of nanofiller dispersion in polymers influences the interfacial interactions ${ }^{13}$, including hydrogen bonding, van der Waals attractions and/or electrostatic forces between the polymer and filler in the nanocomposites ${ }^{14}$. The interfacial interactions between the polymer and filler further determine the stress transfer ${ }^{15,16}$. Therefore, exploiting the properties of the polymer chains adsorbed on the nanofiller surface and quantitative evaluation of the interphase zone arise as important issues ${ }^{6,17}$.

Barroso-Bujans and co-workers ${ }^{18}$ investigated poly(ethylene oxide) (PEO) adsorption onto GO and reduced graphene oxide (RGO). The study observed that the amount of adsorbed PEO on the surface of GO nanosheets was less than 1 wt\% and that no diffraction features were observed that can be assigned to the crystalline phase of PEO. The same group ${ }^{19}$ also reported the effect of GO oxidation degree and thermal exfoliation of RGO on the uptake of PEO. The amount of PEO intercalated in GO increased by increasing the GO oxidation degree. A clear glass transition of the PEO appeared at -64.1 ${ }^{\circ} \mathrm{C}$ and the melting transition at $30.8^{\circ} \mathrm{C}$ in the case with pristine graphene. However, PEO was unable to either crystallize or display a glass or melting transition with $\mathrm{GO}$ and RGO from differential scanning calorimetry ${ }^{18,19}$. Subsequently, the researchers ${ }^{20}$ investigated the effect of the reduction degree of GO on the PEO adsorption, and obtained the amounts of intercalated PEO in the samples of 27, 21 and 2 $w t \%$ for PEO/GO, PEO/partial reduced GO (pRGO) and PEO/RGO, respectively. By using different chain sizes of poly(ethylene glycol) (PEG), the group ${ }^{21}$ further confirmed that restricting the polymer by GO led to a significant reduction in vibrational motions of polymer chains. These investigations ${ }^{18-21}$ show clear evidence of the disappearance and complete suppression of the glass and melting transitions of PEO phase on GO, pRGO and RGO nanosheets of PEO/GO, PEO/pRGO, PEO/RGO from DSC results, despite using different GO types and polymer chain sizes. Similar PEO restrictions were also obtained for other particles such as clays ${ }^{22-24}$, silica ${ }^{25}$ and oxides $^{26}$. In spite of various studies ${ }^{18-21,27}$ focusing on the effect of the starting material, such as GO, RGO or the polymer, on the structure and physical properties of the adsorbed polymer, understanding the effects of processing condition on the structure and adsorption behaviour of the polymer in polymer-GO nanocomposites is still limited.

This project aims to investigate the structure-property relationships of polymer-GO nanocomposites, in particular in relation to the interfacial regions, and obtain a better understanding in this filed. A 
semicrystalline polymer, PEG, with various molecular weights, was selected as the model polymer to investigate the effect of $\mathrm{GO}$ on the adsorbed polymer compared to the bulk polymer. PEG is a hydrophilic polymer available in a broad range of molecular weights. It is widely used as a polymer model for adsorption and nanocomposite studies ${ }^{28}$. Further, GO reinforced PEG may have potential in various applications, such as drug delivery ${ }^{29}$, cancer treatment ${ }^{30}$ and energy conversion and storage ${ }^{31}$. Protocols with different parameters including different mixing times, washing procedures, mixing ratios of materials and molecular weights of PEG, were applied to study their effect on the adsorption behaviour of PEG onto GO nanosheets and hence optimise the adsorption. These important factors have not been systematically addressed before, and could offer a better understanding of the PEG adsorption behaviour and reduce this gap of knowledge whilst also providing guidance in manipulating the properties, such as mechanical and thermal properties, of polymer/GO nanocomposites. GO was firstly prepared, then mixed with the PEGs in water for polymer adsorption. The adsorption amount, structure and physical properties of PEG/GO hybrid nanomaterials were intensively investigated using various characterization techniques.

\section{EXPERIMENTAL SECTION}

\section{Materials}

Graphite powder $(\leq 20 \mu \mathrm{m})$, potassium permanganate $\left(\mathrm{KMnO}_{4}\right)$, sodium nitrate $\left(\mathrm{NaNO}_{3}\right)$, hydrogen peroxide, sulfuric acid $\left(\mathrm{H}_{2} \mathrm{SO}_{4}\right.$ ) (analytical grade, 99\%), hydrochloric acid $(\mathrm{HCl}, 35 \%$ ) and poly(ethylene glycol)s with different molecular weights (Mw) of 960, 10000 and 100000 were purchased from SigmaAldrich Company, UK. It is noted that PEGs of higher molecular weights should be termed as PEOs ${ }^{32}$. For simplicity, PEG is used for both PEGs and PEOs in this work.

\section{Synthesis and Purification of Graphene Oxide}

Graphite oxide was synthesized from graphite powder by a modified Hummer's method ${ }^{33}$. The resulting graphite oxide was washed multiple times with $\mathrm{HCl}$ and distilled water at 1:4 a ratio, followed by distilled water alone, to remove metal ions and other impurities ${ }^{34}$, dried in a freeze dryer and stored in a desiccator before use.

\section{Surface adsorption of PEG onto GO sheets}

Various formulations and methods were used to prepare PEG/GO nanosheets including different mixing times, molecular weights, material ratios and washing procedures. Firstly, the PEG was dissolved in distilled water with concertation of $4.2 \mathrm{wt} / \mathrm{vol} \%$. The GO was dispersed with a concentration of $0.1 \mathrm{wt} / \mathrm{vol} \%$ in distilled water. Both the PEG solution and the GO suspension at a predetermined ratio were mixed together at room temperature using a magnetic stirrer for different periods of time. Then, the mixed PEG/GO suspensions were centrifuged at $8000 \mathrm{rpm}$ for $30 \mathrm{~min}$ using 
an Eppendorf 5804 centrifuge to remove excess PEG in the supernatant, with the sediments collected (denoted as non-washed samples). Part of the sediments was further washed with distilled water for $30 \mathrm{~min}$ by magnetic stirring, followed by centrifugation at $8000 \mathrm{rpm}$ for $30 \mathrm{~min}$ to remove loosely bound PEG and collect the sediments (denoted as washed samples). All the collected samples were dried for 15 hours at $40{ }^{\circ} \mathrm{C}$ in a vacuum oven under $0.1 \mathrm{MPa}$ pressure until the mass of samples became constant. PEG samples without GO were prepared in parallel as controls. The samples were kept in a desiccator under vacuum. The samples prepared are listed in Table 1. Letters $\mathrm{N}, \mathrm{h}$ and $\mathrm{w}$ in the sample codes refer to nanomaterials of PEG/GO, mixing hour and number of washing time. Unless otherwise stated, the molecular weight of the polymer was 10k, the mixing ratio of PEG:GO was $3: 1$ and the washing time was 2 . For example, N72h means the mixing hour was 72 hours, and N100k-1.5-24h means the molecular weight of PEG was 100k, the mixing ratio of PEG:GO was 1.5 and the mixing time was 24 hours.

Table 1. The preparation methods and sample denotations of PEG/GO hybrid nanomaterials.

\begin{tabular}{l|ccccc}
\hline Sample ID & $\begin{array}{c}\text { Polymer Molecular } \\
\text { Weight }\end{array}$ & \multicolumn{2}{c}{ Mixing Ratio } & Mixing & Washing \\
\cline { 3 - 4 } & PEG & GO & Time / h & Number \\
\hline N1h & $10 k$ & 3 & 1 & 1 & 2 \\
N3h & $10 k$ & 3 & 1 & 3 & 2 \\
N6h & $10 k$ & 3 & 1 & 6 & 2 \\
N12h & $10 k$ & 3 & 1 & 12 & 2 \\
N24h & $10 k$ & 3 & 1 & 24 & 2 \\
N72h & $10 k$ & 3 & 1 & 72 & 2 \\
N144h & $10 k$ & 3 & 1 & 144 & 2 \\
N192h & $10 k$ & 3 & 1 & 192 & 2 \\
N1k-1.5-24h & $1 k$ & 1.5 & 1 & 24 & 2 \\
N10k-1.5-24h & $10 k$ & 1.5 & 1 & 24 & 2 \\
N100k-1.5-24h & $100 k$ & 1.5 & 1 & 24 & 2 \\
N-1.5-192h & $10 k$ & 1.5 & 1 & 192 & 2 \\
N-1.5-192h-0w & $10 k$ & 1.5 & 1 & 192 & 0 \\
N192h-0w & $10 k$ & 3 & 1 & 192 & 0 \\
N72h-0w & $10 k$ & 3 & 1 & 72 & 0 \\
N24h-0w & $10 k$ & 3 & 1 & 24 & 0 \\
\hline
\end{tabular}

\section{Characterization}

Fourier transform infrared spectroscopy (FTIR) with a resolution of $1 \mathrm{~cm}^{-1}$ was performed using a Perkin Elmer Spectrum 100 with the attenuated total reflectance in the wavenumber region of 4000 to $400 \mathrm{~cm}^{-}$ ${ }^{1}$. X-ray diffraction (XRD) was achieved using a Stoe Stadi P $\mathrm{CuK}_{\alpha 1}$ with an irradiation wavelength of 0.154 $\mathrm{nm}$, from $2-100^{\circ}(2 \theta)$ with a scanning speed of $10^{\circ} \mathrm{min}^{-1}$ at $40 \mathrm{kV}$ and $35 \mathrm{~mA}$.

Thermogravimetric analysis (TGA) was performed on a Perkin Elmer Pyris 1 TGA. The samples were heated from 25 to $600{ }^{\circ} \mathrm{C}$ with a rate of $10{ }^{\circ} \mathrm{C} \mathrm{min}^{-1}$ under nitrogen gas at a flow rate of $20 \mathrm{ml} \mathrm{min}$. 
Differential scanning calorimetry (DSC) was carried out on a Perkin Elmer DSC6 with two consecutive heating/cooling cycles from $30{ }^{\circ} \mathrm{C}$ to $90{ }^{\circ} \mathrm{C}$, at a rate of $10{ }^{\circ} \mathrm{C} \mathrm{min}-1$ under the flow of nitrogen gas as a rate of $20 \mathrm{ml} \mathrm{min}^{-1}$. The second heating cycle was used for analysis.

Optical microscopy (OM) was performed using a Nikon Eclipse LV150 microscope to reveal PEG crystalline structure on the GO nanosheets. The GO was dispersed in distilled water using a magnetic stirrer for $24 \mathrm{~h}$ and a sonication bath for 30 minutes. A few drops of $\mathrm{GO}$ suspension were placed on a glass substrate and left in the air to dry for overnight at room temperature. The PEG was melted on the GO using a vacuum oven at $80{ }^{\circ} \mathrm{C}$ for an hour. Scanning electron microscopy (SEM) was conducted on an FEl inspect $\mathrm{F}$ scanning electron microscope at voltage $5 \mathrm{kV}$. Samples were placed on an aluminium stub and coated using a gold sputter coater (Emscope SC500A). Atomic force microscopy (AFM) was conducted using a Veeco Dimension 3100 atomic force microscope with an Olympus AC160TS probe with tapping mode at $0.5 \mathrm{~Hz}$. One or two drops of the $\mathrm{GO}$ suspension prepared above were placed on a freshly cleaved mica surface which was left for air-drying overnight.

\section{RESULTS AND DISCUSSION}

\section{Characterization of GO}
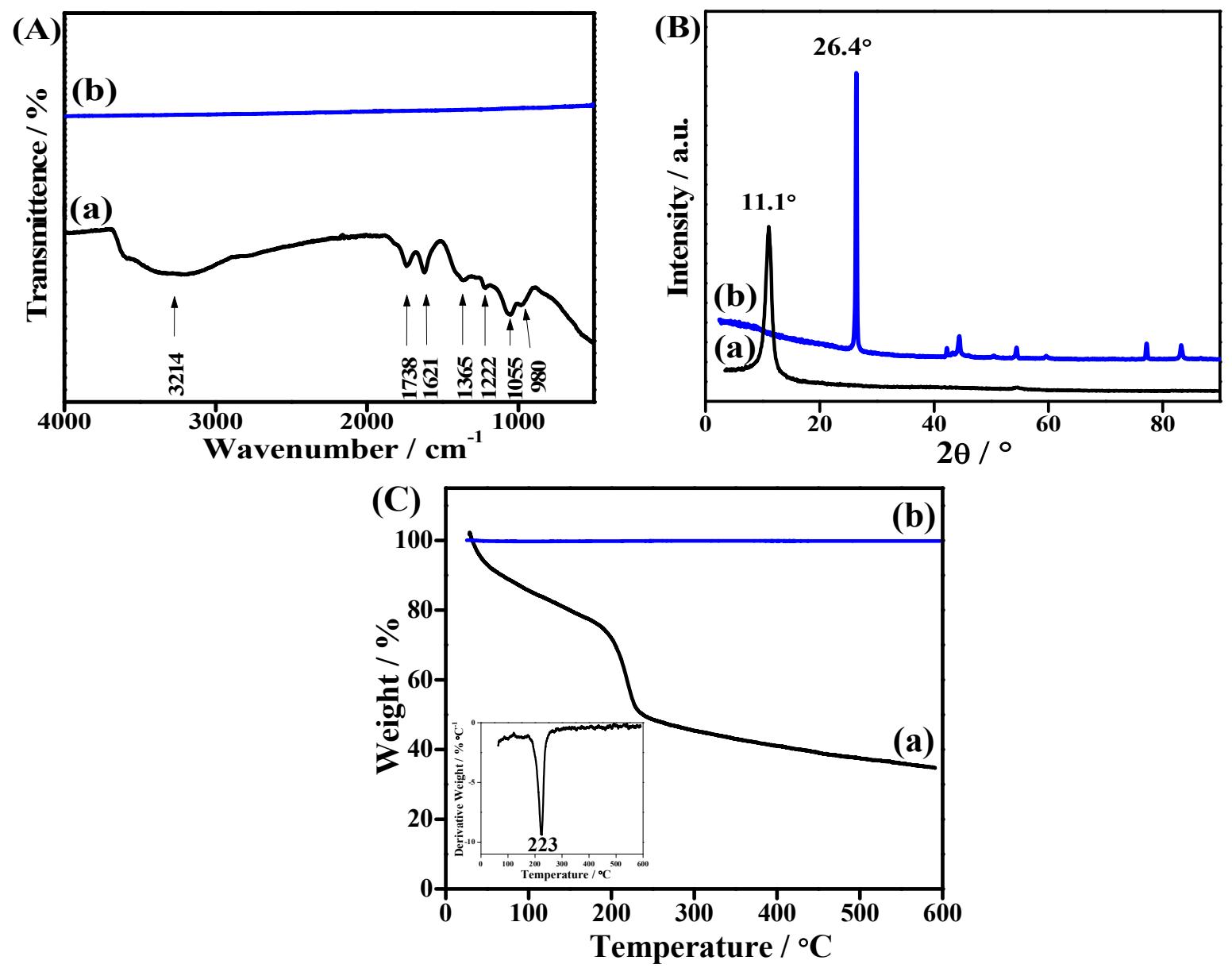
Figure 1. (A) FTIR spectra, (B) XRD traces, and (C) TGA curves of (a) GO and (b) graphite.

Figure 1 shows the FTIR, XRD and TGA results of the GO nanosheets. In Figure 1(A), GO nanosheets showed new characteristic FTIR peaks at 3214, 1738, 1621, 1365, 1222, 1055 and $980 \mathrm{~cm}^{-1}$ wavenumbers corresponding to $\mathrm{O}-\mathrm{H}$ (free water) stretching, carbonyl $\mathrm{C}=\mathrm{O}$ stretching, aromatic ring $\mathrm{C}=\mathrm{C}$ stretching, $\mathrm{O}$ $\mathrm{H}$ bending, $\mathrm{C}-\mathrm{O}$ stretching, epoxy $\mathrm{C}-\mathrm{O}-\mathrm{C}$ stretching and $\mathrm{C}-\mathrm{O}$ stretching functional groups, respectively ${ }^{33,35}$. In Figure $1(B)$, a sharp XRD peak of graphite was presented at $2 \theta=26.4^{\circ}$ corresponding to the (002) peak with an interlayer spacing of $0.33 \mathrm{~nm}$, in agreement with the literature value ${ }^{18,35}$. The (001) peak of $\mathrm{GO}$ appears at $2 \theta=11.1^{\circ}$ during the strong oxidation of graphite to synthesise $\mathrm{GO}$, indicating an interlayer spacing of $0.79 \mathrm{~nm}$, which is in line with other studies ${ }^{33,35}$. Figure $1(\mathrm{C})$ shows the thermal degradation of $\mathrm{GO}$ that constituted $31 \mathrm{wt} \%$ of the total dry mass between $150-250{ }^{\circ} \mathrm{C}$, due to the degradation of epoxide, hydroxyl, carbonyl and carboxyl functional groups ${ }^{35}$. The peak degradation temperature of the $\mathrm{GO}$ was located at $223^{\circ} \mathrm{C}$ as shown in Figure $1(\mathrm{C})$. These characterization results confirmed that the GO nanosheets were successfully prepared.

The Effect of Mixing Time
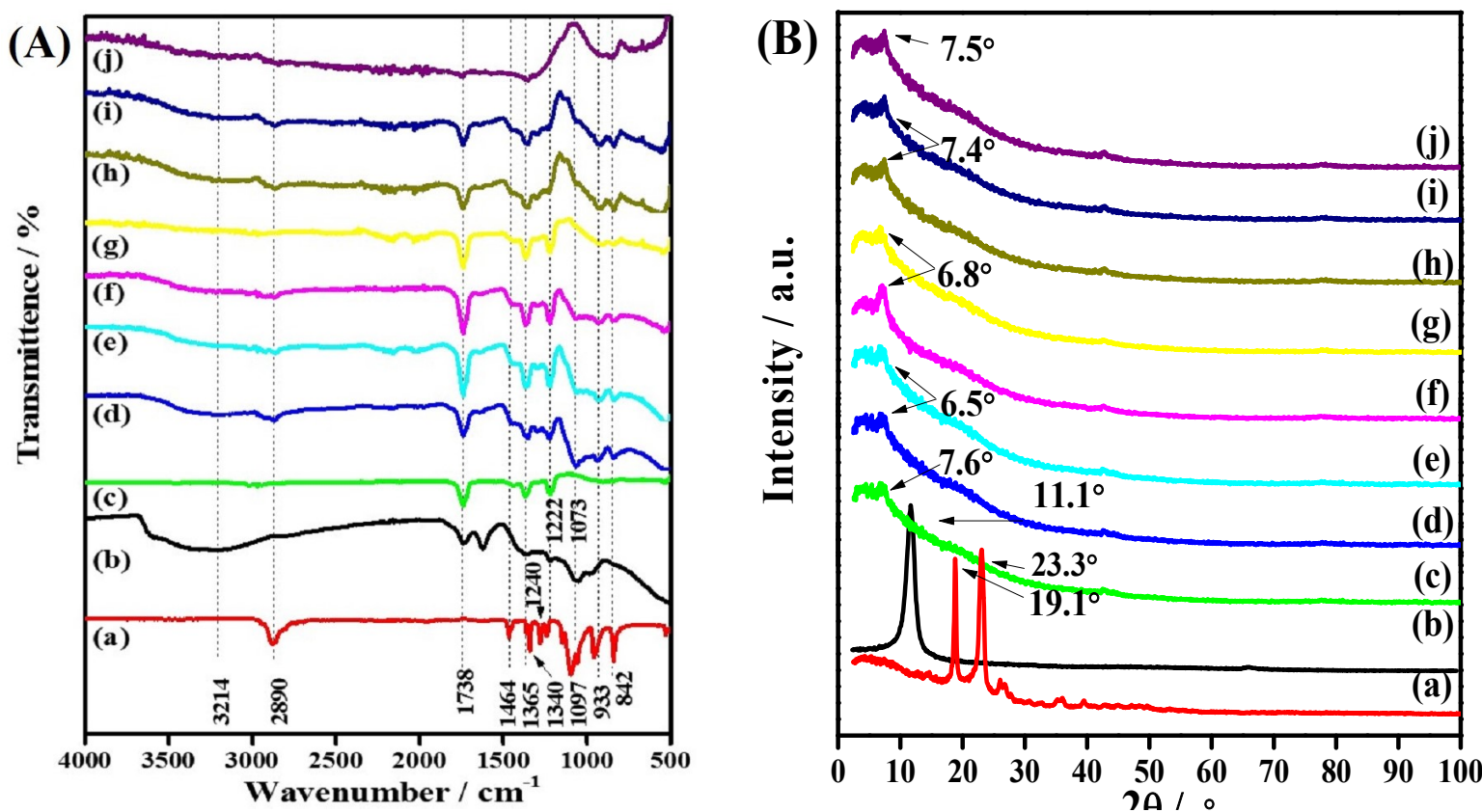

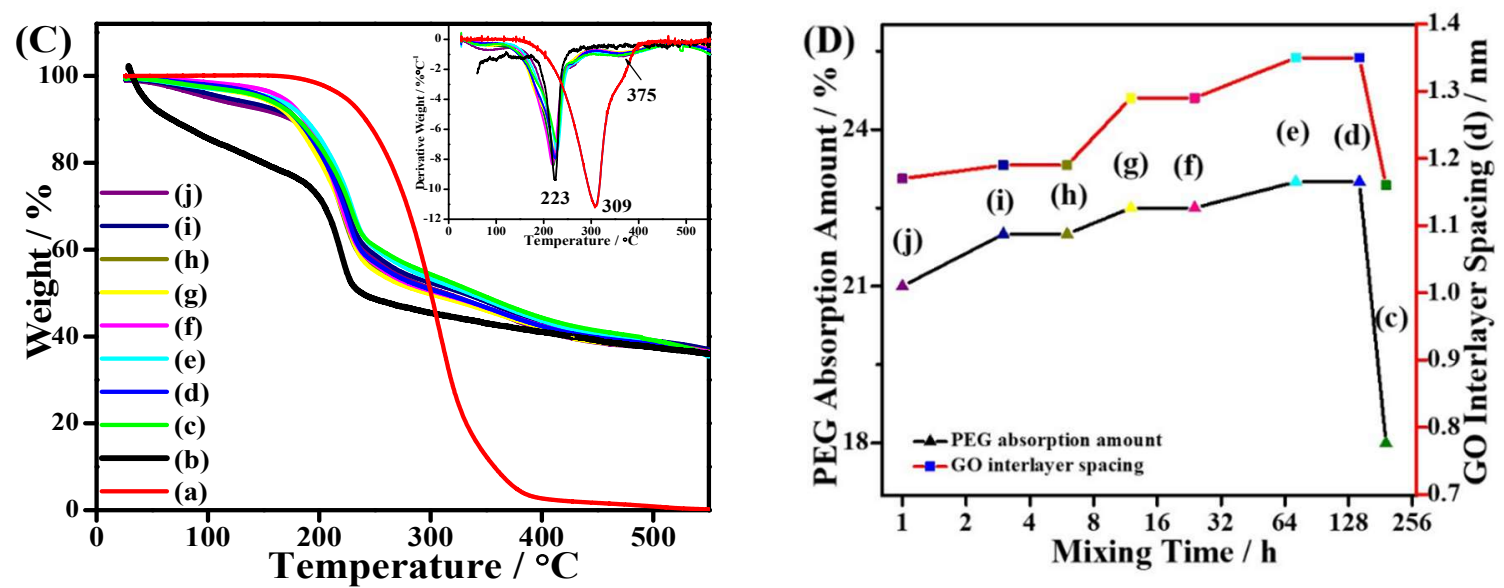

Figure 2. (A) FTIR spectra, (B) XRD traces, (C) TGA curves and (D) the PEG absorption amount and GO interlayer spacing for various mixing times of (a) 10k PEG, (b) GO, and hybrid nanomaterials: (c) 192h, (d) $144 \mathrm{~h},(\mathrm{e}) 72 \mathrm{~h},(\mathrm{f}) 24 \mathrm{~h},(\mathrm{~g}) 12 \mathrm{~h},(\mathrm{~h}) 6 \mathrm{~h},(\mathrm{i}) 3 \mathrm{~h}$ and (j) $1 \mathrm{~h}$.

A series of $P E G / G O$ hybrid nanomaterials with different mixing times were prepared to investigate the effect of mixing time on the adsorption behaviour of the PEG onto GO nanosheets. Figure 2 shows the samples that were prepared by mixing the 10k PEG with GO using 3:1 as the ratio for eight different mixing times in the range from $1 \mathrm{~h}$ to $192 \mathrm{~h}$, with two wash procedures. In Figure 2(A), the FTIR spectrum of the PEG showed characteristic absorption peaks, which were $\mathrm{C}-\mathrm{H}$ stretching at $2890 \mathrm{~cm}^{-1}, \mathrm{C}-\mathrm{H}$ bending at 1464 and $1340 \mathrm{~cm}^{-1}, \mathrm{C}-\mathrm{O}-\mathrm{C}$ stretching at $1097 \mathrm{~cm}^{-1}, \mathrm{C}-\mathrm{O}$ stretching at $933 \mathrm{~cm}^{-1}$ and C-H stretching at $842 \mathrm{~cm}^{-136}$. The strong absorption peaks from GO were presented at $1738 \mathrm{~cm}^{-1}, 1365 \mathrm{~cm}^{-1}$ and $1222 \mathrm{~cm}$ ${ }^{1}$ of $\mathrm{C}=\mathrm{O}, \mathrm{O}-\mathrm{H}$ and $\mathrm{C}-\mathrm{O}$, respectively, in the hybrid samples. The intermolecular hydrogen bonding between the ether group such as $\mathrm{C}-\mathrm{O}-\mathrm{C}$ sites of PEG chain interacts with the polar functional groups of $\mathrm{GO}$, such as $\mathrm{COOH}$ and $-\mathrm{OH}^{37}$; for instance, the peak of $\mathrm{C}-\mathrm{O}-\mathrm{C}$ stretching in PEG shifted from 1097 to $1073 \mathrm{~cm}^{-1}$ in agreement with literature ${ }^{38}$, where hydrogen bonding is considered the major factor in the interactions between the GO nanosheets and PEG, which led to an improvement in the PEG adsorption behaviour ${ }^{39}$. These results confirmed the adsorption of the PEG and their interfacial interactions with the GO nanosheets, which clearly presented in all hybrid nanomaterials except the N1h sample. This may imply a low ratio of polymer adsorption on GO nanosheets with shorter mixing time (1h), whereas the absorption peaks of PEG exhibited higher increase in the intensity with increasing mixing time from $3 \mathrm{~h}$ to $72 \mathrm{~h}$. This suggested improvement in the PEG amount in the hybrids, whereas the intensity of the absorption peaks decreased after increasing mixing time from $72 \mathrm{~h}$ to $192 \mathrm{~h}$ (which was the longest mixing time in this study). This indicated a reduction in the adsorbed polymer in the latter.

Figure 2(B) shows the XRD peaks of PEG, with (120) and (032) peaks appearing at $2 \theta=19.1^{\circ}$ and $2 \theta=23.5^{\circ}$ usually reported in the literature ${ }^{40}$. The (001) peak of GO shifted from $2 \theta=11.1^{\circ}$ to $7.5^{\circ}, 7.4^{\circ}$, 
$7.4^{\circ}, 6.8^{\circ}, 6.8^{\circ}, 6.5^{\circ}, 6.5^{\circ}$ and $7.6^{\circ}$ in the hybrid nanomaterials suggesting the interlayer spacing of $\mathrm{GO}$ has increased from $0.79 \mathrm{~nm}$ to $1.17,1.19,1.19,1.29,1.29,1.35,1.35$ and $1.16 \mathrm{~nm}$ for N1h, N3h, N6h, N12h, N24h, N72h, N144h and N192h, respectively. This means the PEG has intercalated (or been absorbed) into the interlay spacing of GO nanosheets, and an increased amount of intercalating PEG might have increased the interlayer spacing. The interlayer spacing increased by $0.18 \mathrm{~nm}$ with an increased mixing time from $1 \mathrm{~h}$ to $144 \mathrm{~h}$, whereas it decreased by $0.19 \mathrm{~nm}$ with an increased mixing ratio from $144 \mathrm{~h}$ to $192 \mathrm{~h}$. This is in agreement with FTIR results as discussed earlier. Figure 2(C) shows the thermal degradation behaviour of PEG, GO and their hybrid nanomaterials. The PEG was thermally stable in the range of $30-170^{\circ} \mathrm{C}$. Between $200-400{ }^{\circ} \mathrm{C}$, the mass of PEG lost $79 \mathrm{wt} \%$ then up to $99 \mathrm{wt} \%$ at $550^{\circ} \mathrm{C}$ due to the degradation of the PEG backbone. The mass of hybrid nanomaterials was reduced by 39 wt\% between $150-250{ }^{\circ} \mathrm{C}$, which was related to the degradation of the oxygenated functional groups of the GO. Moreover, between $250-420^{\circ} \mathrm{C}$, the samples lost $19 \mathrm{wt} \%$ of its mass attributable to the degradation of the polymer in hybrids. The total loss of the hybrid mass was $65 \mathrm{wt} \%$ at $550^{\circ} \mathrm{C}$ due to the presence of $\mathrm{GO}$ which induces barrier effects ${ }^{41}$ and hydrogen bonding with the polymer. The peak degradation temperatures $\left(T_{d}\right)$ were located at 223 and $309{ }^{\circ} \mathrm{C}$ of $\mathrm{GO}$ and PEG, respectively (Figure $2 \mathrm{C}$ ). These $T_{d}$ values were shifted in the hybrids. First, the $T_{d}$ of GO shifted to $224-229{ }^{\circ} \mathrm{C}$ in the hybrids, whereas the second $T_{d}$ of PEG improved notably to $372-378{ }^{\circ} \mathrm{C}$. The incorporation of GO improved the non-oxidative thermal stability of the PEG in the hybrids compared to that of the neat PEG, in agreement with the literature ${ }^{42}$.

Figure 2(D) illustrates the absorption amounts of PEG from TGA and the interlayer spacing of GO nanosheets in the hybrids from XRD, as a function of mixing time. The absorbed amount of PEG in the PEG/GO nanohybrids, $a$, was calculated using equation $(1)^{43}$.

$a=1-\frac{R_{h y b r i} /\left(1-M_{h y b}\right)}{R_{G O} /\left(1-M_{G O}\right)}$

where $R_{\text {hybrid }}$ and $R_{G O}$ are the residual amounts of the hybrid and $G O$ at $550{ }^{\circ} \mathrm{C}$ respectively and $M_{\text {hybrid }}$ and $\mathrm{M}_{\mathrm{GO}}$ are their moisture contents at $100{ }^{\circ} \mathrm{C}$. The residual amount of PEG at $550{ }^{\circ} \mathrm{C}$ was approaching zero so was neglected. The absorption amount of PEG in the samples increased slightly from 21 to 23 wt\% with an increase in the mixing time from $1 \mathrm{~h}$ to $144 \mathrm{~h}$ and was reduced to $18 \mathrm{wt} \%$ for the $192 \mathrm{~h}$ mixing time. The results confirmed an increase in the interlayer spacing of $\mathrm{GO}$ nanosheets was due to an increased amount of the intercalated polymer in the samples. The reduced absorption amount at $192 \mathrm{~h}$ suggested that the PEG intercalated via weak physical bonds, which could be removed by the long mixing time. 

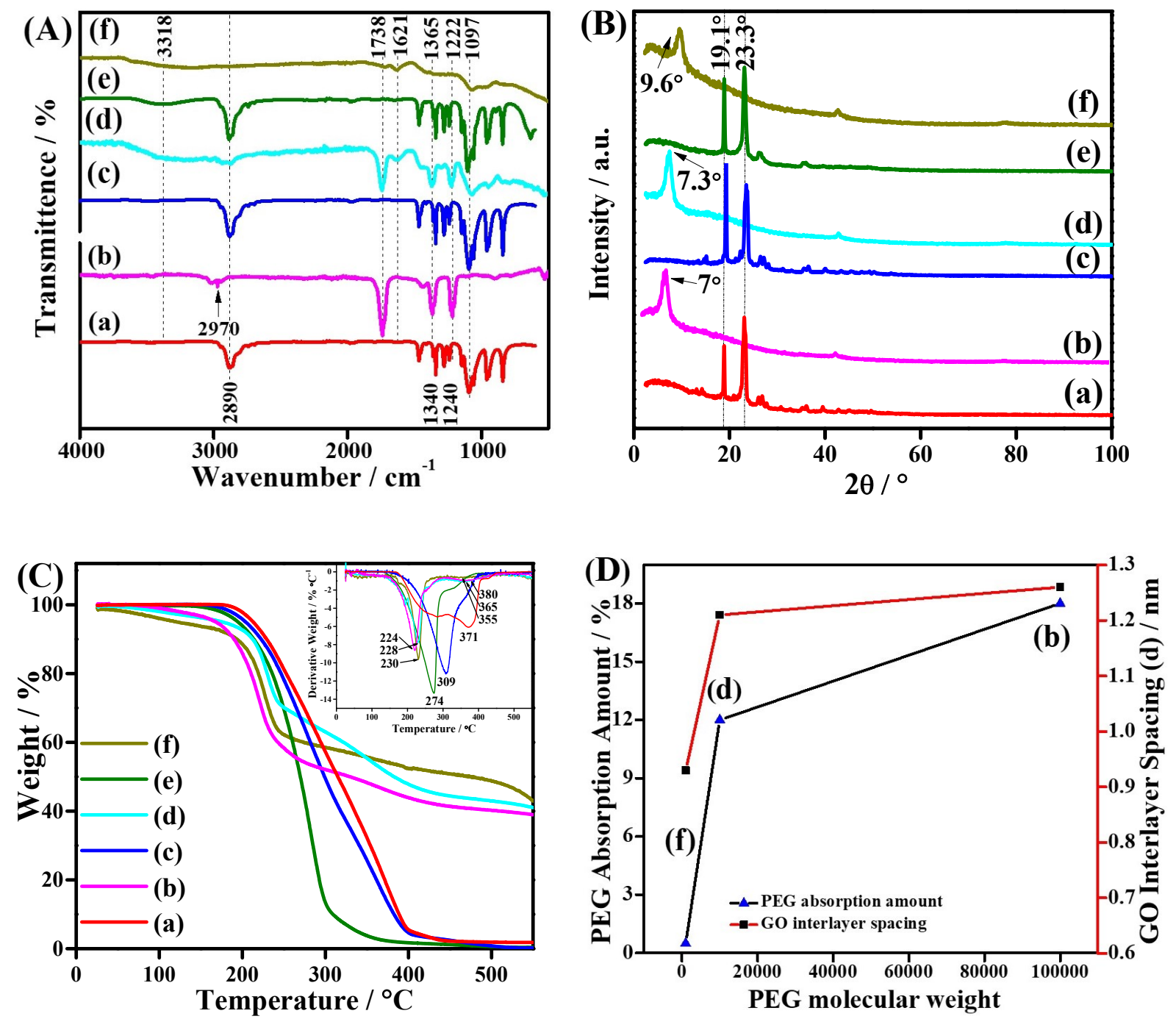

Figure 3. (A) FTIR spectra, (B) XRD traces, (C) TGA curves, and (D) PEG absorption amounts and GO interlayer spacing of (a) 100k PEG, (b) N100k-1.5-24h, (c) 10k PEG, (d) N10k-1.5-24h, (e) 1k PEG and (f) N1k-1.5-24h hybrids.

Figure 3 shows the effect of polymer molecular weight on the adsorption behaviour of PEG onto the GO nanosheets. Three different molecular weights, i.e. 1k, 10k and 100k, of PEG were used for preparing three PEG/GO hybrids, N1k-1.5-24h, N10k-1.5-24h and N100k-1.5-24h. Figure 3(A) shows the FTIR spectra of the PEGs and hybrids. N1k-1.5-24h showed the same peaks of the functional groups of GO, with only small features of $1 \mathrm{k}$ PEG, whereas N10k-1.5-24h and N100k-1.5-24h presented the strong absorption peaks from both PEGs and GO. For instance, the C-H stretching of PEG shifted from 2890 to $2970 \mathrm{~cm}^{-1}$ for the N100k-1.5-24h. The hydrogen bonding between C-O-C groups of PEG chains and $\mathrm{COOH}$ or $-\mathrm{OH}$ of GO nanosheets led to a shift of C-O-C peak, e.g. from 1097 to $1067 \mathrm{~cm}^{-1}$ in N1k-1.5-24h hybrid. Figure $3(B)$ shows the XRD traces of the hybrids. The (001) peak of GO shifted from $2 \theta=11.1^{\circ}$ to 
$9.6^{\circ}, 7.3^{\circ}$ and $7.0^{\circ}$ corresponding to increases in the interlayer spacing from $0.79 \mathrm{~nm}$ to $0.93,1.21$ and $1.26 \mathrm{~nm}$ for N1k-1.5-24h, N10k-1.5-24h and N100k-1.5-24h hybrids, respectively. These shifts implied the intercalation of the PEG into GO nanosheets. Generally, it was observed that the hybrids, which contained higher molecular weights, showed a higher intensity for the (001) peak and larger interlayer spacing compared with those hybrids containing lower molecular weight PEGs. These results suggested a higher intercalation amount of the polymer ${ }^{21,44}$, in agreement with the FTIR results discussed above. The hydrogen bonding between the functional groups of GO and PEG helped the intercalation of the PEG in between GO nanosheets.

The TGA curves in Figure 3(C) showed more mass loss for the hybrids that contained 10k and 100k PEG compared to the N1k-1.5-24h with 1k PEG. The losses of mass were 31.7, 38.3 and $26.2 \mathrm{wt} \%$ between 150-250 ${ }^{\circ} \mathrm{C}$, whereas the total mass losses were $38.9,41.1$ and $42.7 \mathrm{wt} \%$ at $550{ }^{\circ} \mathrm{C}$ for N1k-1.5-24h-2w, N10k-1.5-24h-2w and N100k-1.5-24h-2w, respectively. The ratio of mass loss increased by $9.7 \%$ when the molecular weight increased from $1 \mathrm{k}$ to $100 \mathrm{k}$. This finding supports the increase of the interlayer spacing due to the increasing molecular weight of PEG. Each hybrid also presented two peak degradation temperatures, which were higher than the values for the neat GO and PEG. The $T_{d}$ of GO shifted from $223^{\circ} \mathrm{C}$ to 224,228 and $230^{\circ} \mathrm{C}$, whereas the $T_{d}$ of the PEGs shifted from 274,309 and 371 ${ }^{\circ} \mathrm{C}$ to 355,365 and $380^{\circ} \mathrm{C}$ of $1 \mathrm{k}, 10 \mathrm{k}, 100 \mathrm{k}, \mathrm{N} 1 \mathrm{k}-1.5-24 \mathrm{~h}, \mathrm{~N} 10 \mathrm{k}-1.5-24 \mathrm{~h}$ and N100k-1.5-24h, respectively, due to the degradation of the PEG.

Figure 3(D) summarizes the PEG absorption amount between the GO nanosheets and the interlayer spacing between the GO nanosheets, as a function of PEG molecular weight. The PEG absorption amount exhibited a significant increase from $1 \mathrm{wt} \%$ to $16 \mathrm{wt} \%$ then to $18 \mathrm{wt} \%$, when the PEG molecular weight was increased from $1 \mathrm{k}$ to $10 \mathrm{k}$ then $100 \mathrm{k}$ for N1k-1.5-24h-2w, N10k-1.5-24h-2w and N100k-1.5$24 \mathrm{~h}-2 \mathrm{w}$, respectively. These results represented a notable increase by $1700 \%$ from N1k-1.5-24h-2w to N100k-1.5-24h-2w, even when the PEG:GO mixing ratio was reduced from 3 to 1.5, in agreement with the $X R D$ results that indicated increases of the $G O$ interlayer spacing from $0.79 \mathrm{~nm}$ of the neat $\mathrm{GO}$ to $0.93 \mathrm{~nm}, 1.21 \mathrm{~nm}$ and $1.26 \mathrm{~nm}$ of the N1k-1.5-24h-2w, N10k-1.5-24h-2w and N100k-1.5-24h-2w, respectively. Generally, the molecular weight is demonstrated as an important factor for improving the PEG absorption amount, where the free energy in the polymer chain significantly increased with the longer polymer chain to achieve planar conformation ${ }^{21,45}$. Meanwhile, the $\mathrm{GO}$ surface has abundant oxygen functional groups ${ }^{21}$ that provide sites to accommodate PEG chains, which led to hydrogen bonding between the polymer and the oxygenated functional groups of GO nanosheets. Similar conclusions were reached in a study by Nelson and Cosgrove ${ }^{46}$, who found that increasing the molecular weight of PEO led to an increase in the hydrodynamic thickness of the adsorption layer of PEO on laponite clay. 
The Effect of Mixing Ratio of Polymer to GO and Wash Procedure
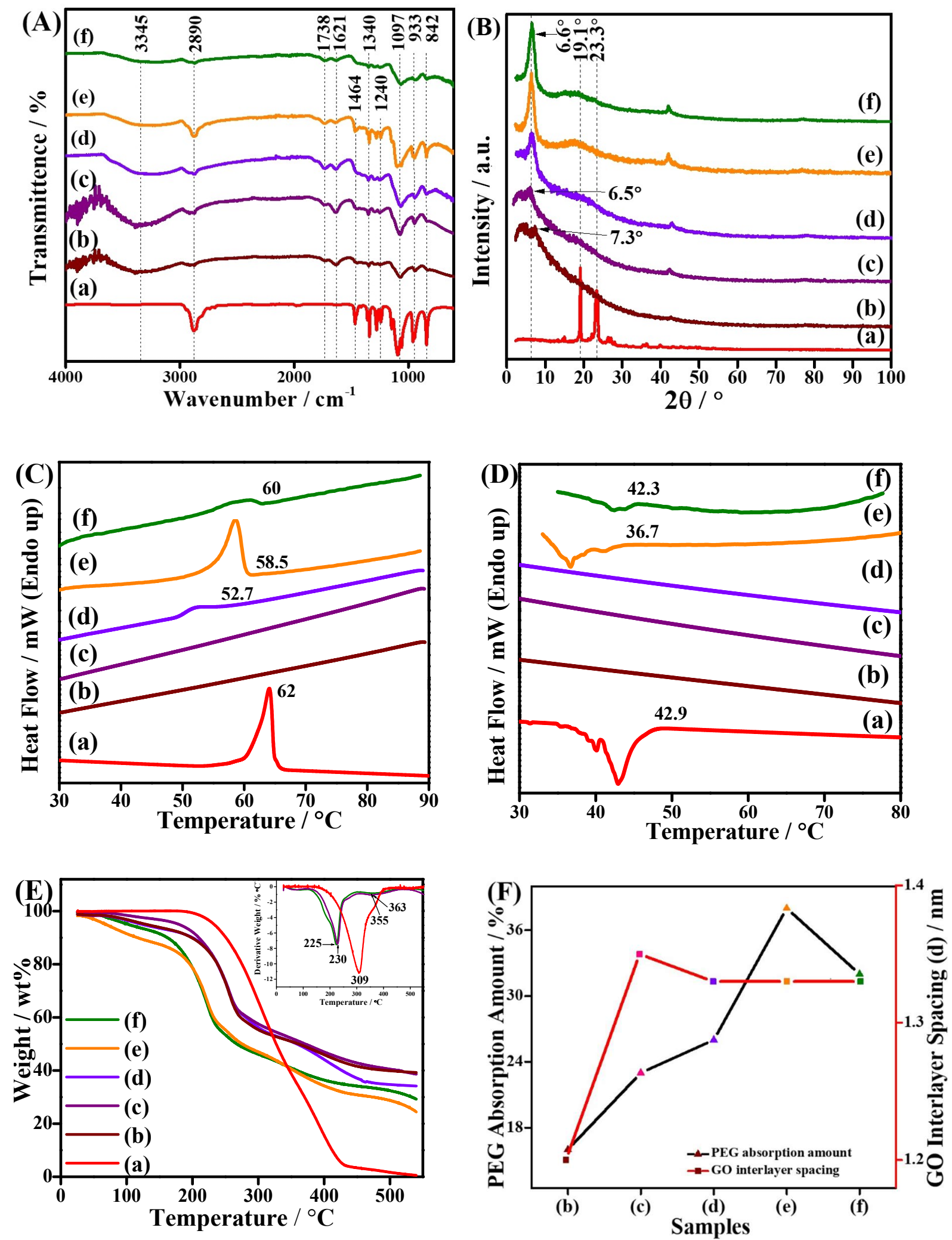

Figure 4. (A) FTIR spectra, (B) XRD traces, (C) DSC heating scan and (D) DSC cooling scan, (E) TGA curves and (F) PEG absorption amounts and GO interlayer spacings of the (a) 10k PEG compared with the (b) N-1.5-192h, (c) N-1.5-192h-0w, (d) N192h-0w (e) N72h-0w and (f) N24h-0w. 
In this section, systematic methods and parameters were applied considering the results from the first two sections. No further washing procedure was applied, the mixing ratio of PEG to GO was increased from 1.5:1 to 3:1, and the mixing time was reduced from 192 to 72 and $24 \mathrm{~h}$, with a fixed PEG molecular weight of 10k, as shown in Table 1.

In Figure 4(A), most functional groups of both $G O$ and PEG were presented in the FTIR spectra of the hybrids. A shift in some PEG peaks could be seen due to the adsorption of PEG on the surface of GO nanosheets compared with those of neat PEG, e.g. the peaks at $940 \mathrm{~cm}^{-1}$ of $\mathrm{C}-\mathrm{O}$ and $833 \mathrm{~cm}^{-1}$ of C-H. Meanwhile, the O-H (free water) absorbtion peak of GO shifted to $3345 \mathrm{~cm}^{-1}$ in the hybrids. The C-O-C peak for PEG shifted to $1073 \mathrm{~cm}^{-1}$ suggesting hydrogen bonding as in the prevous cases. This confirmed the interfacial interactions in these samples. An increase in the adsorption PEG concentration was also found in the N192h-0w and N-1.5-192h-0w compare to their washed samples. Applying no further washing procedure of the samples was an important factor that increased the peak intensity that was related to an increase in the amount of the PEG onto GO nanosheets. The washing procedure was associated with breaking the weak physical bond that attached the PEG on the GO surface. Therefore, no further washing procedure helped keep the PEG adsorbed on the GO nanosheets surface.

Increasing the ratio of PEG from 1.5 to 3 showed an increase in the peak intensity that was clear in the $\mathrm{C}-\mathrm{H}$ bending $\left(1464 \mathrm{~cm}^{-1}\right)$ and $\mathrm{C}-\mathrm{O}$ stretching $\left(1240 \mathrm{~cm}^{-1}\right)$ from PEG, whereas the $\mathrm{C}=\mathrm{O}$ peak $1738 \mathrm{~cm}^{-1}$ of $\mathrm{GO}$ were seen to reduce its intensity for the N192h-0w compared to the N-1.5-192h-0w. The N72h-0w presented the highest intensity of the adsorbed PEG peaks at $1464 \mathrm{~cm}^{-1}$ of C-H bending and $1240 \mathrm{~cm}^{-1}$ of C-O stretching, compared to the spectra of further washed N-1.5-192h hybrids.

Figure 4(B) shows the shifting of the (001) peak of $\mathrm{GO}$ from $2 \theta=11.1^{\circ}$ to $7.3^{\circ}$ of $\mathrm{N}-1.5-192 \mathrm{~h}$. Applying no further washing procedure shifted the peak to $6.5^{\circ}$ corresponding to an increase of the interlayer distancing from $0.79 \mathrm{~nm}$ of $\mathrm{GO}$ to $1.20 \mathrm{~nm}$ then $1.35 \mathrm{~nm}$ for $\mathrm{N}-1.5-192 \mathrm{~h}$ and the no further washed sample $\mathrm{N}-1.5-192 \mathrm{~h}-0 \mathrm{w}$, respectively. The latter interlayer spacing was considered the highest in the current study. The increase in the GO interlayer spacing suggested the increased amount of the confined (intercalated) PEG between the GO nanosheets. Applying no washing procedure meant that the attached polymer on and within GO nanosheets may have been maintained in the hybrid samples.

After reducing the mixing time from 192 to $72 \mathrm{~h}$, then to $24 \mathrm{~h}$, the (001) peak of GO in the hybrid shifted from $11.1^{\circ}$ to $6.6^{\circ}$ corresponding to an increase in the interlayer spacing from 0.79 to $1.33 \mathrm{~nm}$ that showed an increased amount of confined polymer between the GO nanosheets. Further, a new peak appeared between $14^{\circ}-24^{\circ}$ in the sample N192h-0w, which was associated with PEG. This peak of PEG confirmed it had been adsorbed on the surface of GO nanosheets, as the intercalated PEG between the 
GO nanosheets transformed from crystalline to amorphous as further confirmed below. The peak was wider and higher when the mixing time was $72 \mathrm{~h}(\mathrm{~N} 72 \mathrm{~h}-\mathrm{Ow}$ ) compared with $192 \mathrm{~h}$ (N192h-Ow) and $24 \mathrm{~h}$ (N24h-Ow).

Figure 4 (C, D) showed the DSC traces of the PEG and hybrids. In Figure 4 (C), the 10k PEG curve exhibited a sharp melting peak at $62{ }^{\circ} \mathrm{C}$. In the hybrids, the crystalline peak of PEGs disappeared in all samples like in the cases for both the effect of the mixing time and polymer molecular weight sections as shown in Figure S1 (see Supporting Information). Because the long mixing time and washing procedure are associated with removing the adsorbed polymer from the GO nanosheet surface in these hybrids, the 2D-layer GO strongly restricted the intercalated PEG as amorphous phase ${ }^{21}$. Therefore, most of the polymers are confined between the GO nanosheets as absorbed polymers, in agreement with Wang et al. ${ }^{36}$, who reported that XRD crystalline peaks were presented in the PEG/GO hybrids with a ratio of PEG more than $60 \mathrm{wt} \%^{36}$. Also, it matched the finding of Barroso-Bujans and co-authors ${ }^{18-21}$, which showed no sign of melting peaks for the PEO/GO and PEO/RGO hybrid nanomaterials.

A broad melting peak at $52.7^{\circ} \mathrm{C}$ was present after increasing the PEG:GO mixing ratio from $1.5: 1$ to $3: 1$ and applying no washing procedure, which confirmed the adsorption of PEG on the GO nanosheets surface of the N192h-0w. Reducing the mixing time from 192 to $72 \mathrm{~h}$ presented a sharp and significant melting peak at $58.5^{\circ} \mathrm{C}$ of the $\mathrm{N} 72 \mathrm{~h}-\mathrm{OW}$, which showed the biggest melting enthalpy compared to other hybrids. The DSC result of N72h-Ow confirmed the FTIR and XRD results, which showed the higher intensity peaks of the adsorbed PEG. However, further reducing the mixing time to $24 \mathrm{~h}$ exhibited shifting of the melting peak to $60{ }^{\circ} \mathrm{C}$, which was presented as a broad DSC peak of the N24h-Ow. The DSC results of no washing hybrids confirmed the XRD results, which showed the crystalline peak of the adsorption PEG. Generally, the melting peaks of the hybrids shifted toward lower temperature values compared with PEG 10k. The decreasing in the melting temperature $\left(T_{m}\right)$ of the samples was related to the contribution of the high amount of GO nanosheets which restricted the chain motions of the PEG because of the high specific surface area of $\mathrm{GO}$ and the strong interfacial interactions. This led to a reduction in the melting temperatures of the hybrids. A decrease in $T_{m}$ was previously reported in the nanocomposites with an increased graphene ratio ${ }^{19,20}$.

Table 2 shows the $T_{m}$, melting enthalpy $\left(\Delta H_{m}\right)$, crystallisation temperature $\left(T_{c}\right)$, crystallisation enthalpy $\left(\Delta \mathrm{H}_{c}\right)$ and crystallinity. The percentage of crystallinity $\left(\mathrm{X}_{\mathrm{m}}\right)$ of the PEG as bulk or in nanohybrids were calculated from the following equation $(2)^{47}$.

$\mathrm{X}_{\mathrm{c}}=\frac{\Delta \mathrm{H}_{\mathrm{m}}(\mathrm{Tm})}{(1-W) \Delta \mathrm{H}_{\mathrm{m}}^{\mathrm{o}}\left(\mathrm{T}_{\mathrm{m}}^{\mathrm{o}}\right)}$ 
where $\Delta \mathrm{H}_{\mathrm{m}}, \Delta \mathrm{H}_{\mathrm{m}}^{\circ}$ and $W$ are the enthalpic changes associated with melting of the hybrids, the $100 \%$ crystalline of PEO $\left(205 \mathrm{~J} \mathrm{~g}^{-1}\right)^{48}$ and the mass fraction of GO in the hybrid, respectively. The crystallinity of all hybrid samples decreased compared to that of the neat PEG, in agreement with the literature ${ }^{49}$.

Table 2. The DSC results of PEGs and the hybrids.

\begin{tabular}{c|cccc}
\hline Samples Temperature ${ }^{\circ} \mathrm{C}$ & PEG 10k & N192h-0w & N72h-0w & N24h-0w \\
\hline $\mathrm{T}_{\mathrm{m}}$ & 62.2 & 52.7 & 58.5 & 60 \\
$\Delta \mathrm{H}_{\mathrm{m}} \mathrm{J} \mathrm{g}^{-1}$ & 141 & 2.1 & 3.2 & 2.9 \\
$\mathrm{~T}_{\mathrm{c}}$ & 42.9 & - & 36.7 & 42.3 \\
$\Delta \mathrm{H}_{\mathrm{c}} \mathrm{J} \mathrm{g}^{-1}$ & 138 & 0.019 & 0.94 & 0.08 \\
$\mathrm{X}_{\mathrm{c}} \%$ & 69 & 12 & 20 & 14 \\
\hline
\end{tabular}

The thermal degradation temperature of the samples can be seen in Figure 4(E). The first $T_{d}$ of hybrids shifted from $223{ }^{\circ} \mathrm{C}$ of GO to $225^{\circ} \mathrm{C}$ of all the three washed hybrids, whereas it shifted to $230^{\circ} \mathrm{C}$ for nonwashed hybrids. The second $T_{d}$ significantly improved from 309 to $355^{\circ} \mathrm{C}$ and 363 for all the no washing hybrids. The results for the no further washing procedure led to two main important findings: the rapid PEG adsorption reached $24 \mathrm{~h}$ mixing time; and the PEG adsorption amount was considerably enhanced by up to $65.2 \%$ compared to the samples that were washed. The PEG was attached to the GO nanosheets surface with physical bonds. This weak physical interaction was easily lost even with a weak external effect, as previously discussed for the long mixing time $192 \mathrm{~h}$.

Figure 4(F) showed the adsorption amount of the PEG on the GO nanosheets derived from the TGA results in comparison with the interlayer spacing of GO. The results confirmed that most of the PEGs were confined between the GO nanosheets of the samples from N1h to N-1.5-192h-0w. The adsorption amount of PEG exhibited a significant increase from $16 \mathrm{wt} \%$ of $\mathrm{N}-1.5-192 \mathrm{~h}$ to $23 \mathrm{wt} \%$ of N-1.5-192h-Ow without applying washing procedure, whereas increasing the PEG mixing ratio to GO from 1.5:1 to 3:1 of $\mathrm{N} 192 \mathrm{~h}-0 \mathrm{w}$ showed another increase from 23 to $26 \mathrm{wt} \%$ compared to the N-1.5-192h-0w. N192h-0w presented both of the absorbed polymers between the GO nanosheets and the adsorbed polymer on the GO surface at the same time. The polymer adsorption amount was significantly enhanced from 23 to 38 wt\% when reducing the mixing time from 192 to $27 \mathrm{~h}$, but was reduced to $32 \mathrm{wt} \%$ when reducing the mixing time to $24 \mathrm{~h}$.

The attached PEG interacted with the GO nanosheets in two main positions: confined between the GO nanosheets and adsorbed on the GO nanosheets surface. The figure confirmed these two types of PEG attachment. Firstly, the confined PEG between the GO nanosheets showed an increase in the interlayer spacing of GO and the hybrid presented a higher amount of PEG. Secondly, the adsorbed PEG on the GO nanosheets showed the crystalline and the melting peaks. According to these findings, the PEG in the 
washed samples was confined between the GO nanosheets, whereas the other samples presented two attaching types of PEG.
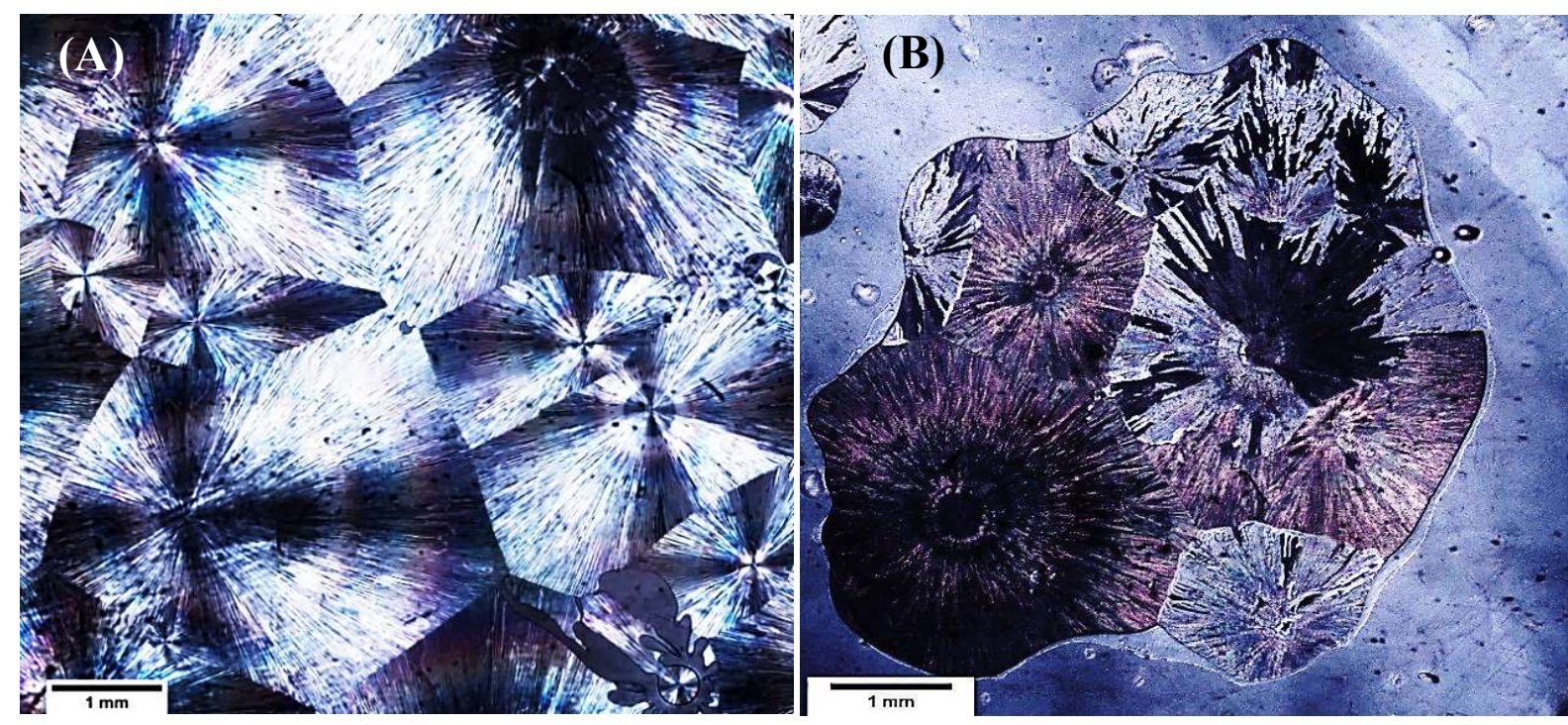

Figure 5. Polarised optical microscopy images of (A) PEG 10k and (B) N72h-0w.

The effect of GO on the PEG crystalline behaviour is an important issue that was further investigated by using the sample of N72h-Ow hybrid, as shown in Figure 5. The POM images clearly indicate that the neat PEG crystallite size was larger than the PEG on GO nanosheets. Nucleation and crystal growth are the two stages of the crystal formation that are influenced by GO nanosheets. These GO nanosheets are effective nucleating agents on the growth rate of the crystallization and polymer ${ }^{50}$. The significant influence of the interfacial interaction of GO nanosheets could also affect the PEG crystallization. Tong et al. ${ }^{51}$ reported that the PEG spherulite growth rate was slower during the crystallization of polymers on the surface of GO nanosheet. The load transferring efficiency from the semicrystalline polymer to the filler in the bulk polymer nanocomposites depended on the interphase interaction between the filler and matrix properties ${ }^{52}$. These results supported the DSC results, which showed a reduction in the melting from $62{ }^{\circ} \mathrm{C}$ to $58{ }^{\circ} \mathrm{C}$ and the crystallinity from $69 \%$ to $20 \%$ of $\mathrm{N} 72 \mathrm{~h}-0 \mathrm{w}$, compared to neat PEG. A similar phenomenon to that of $\mathrm{GO} /$ polyurethane composites was reported by Cai et al. ${ }^{49}$, where the crystal size of polyurethane was smaller, with the incorporation of GO. 

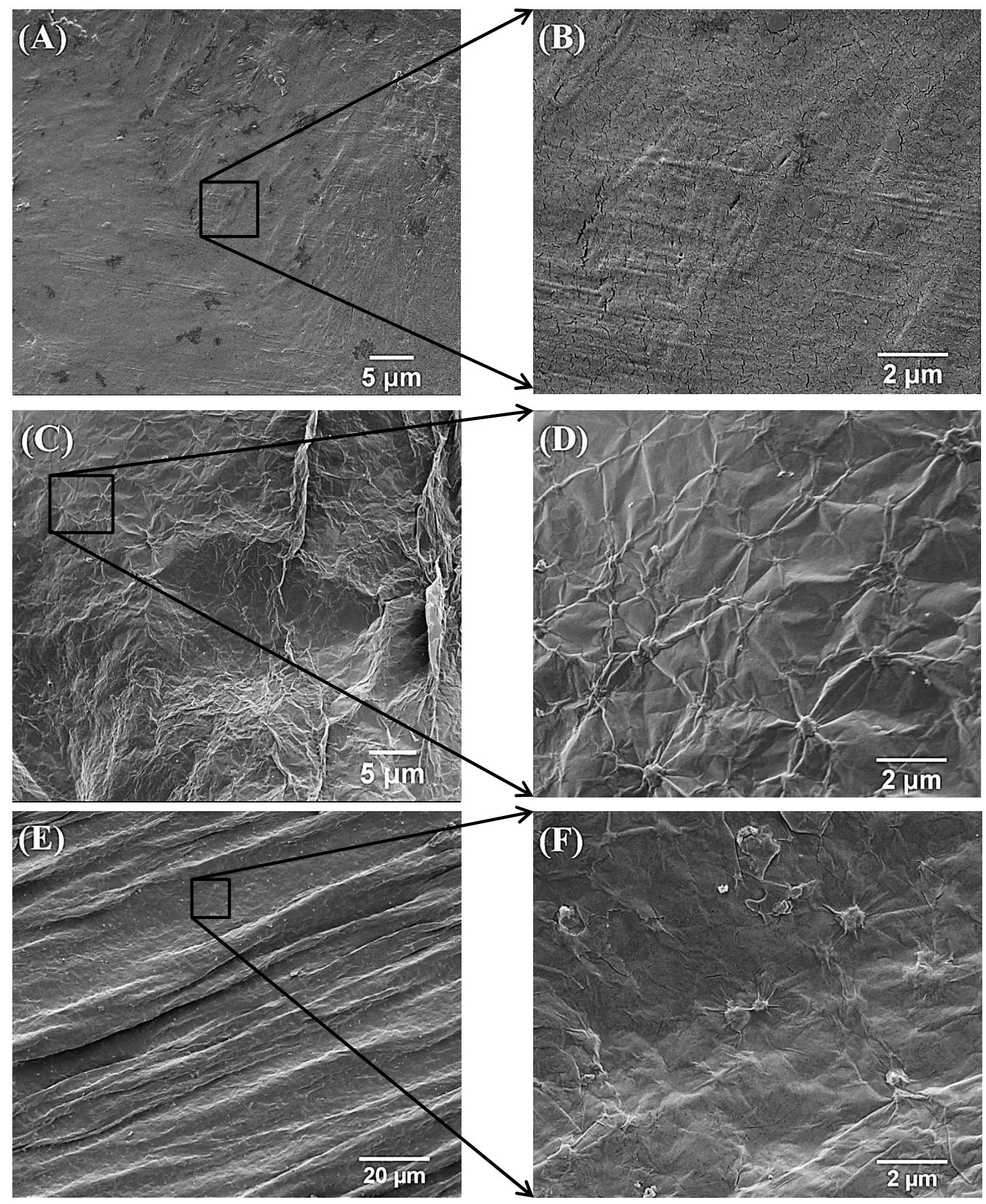

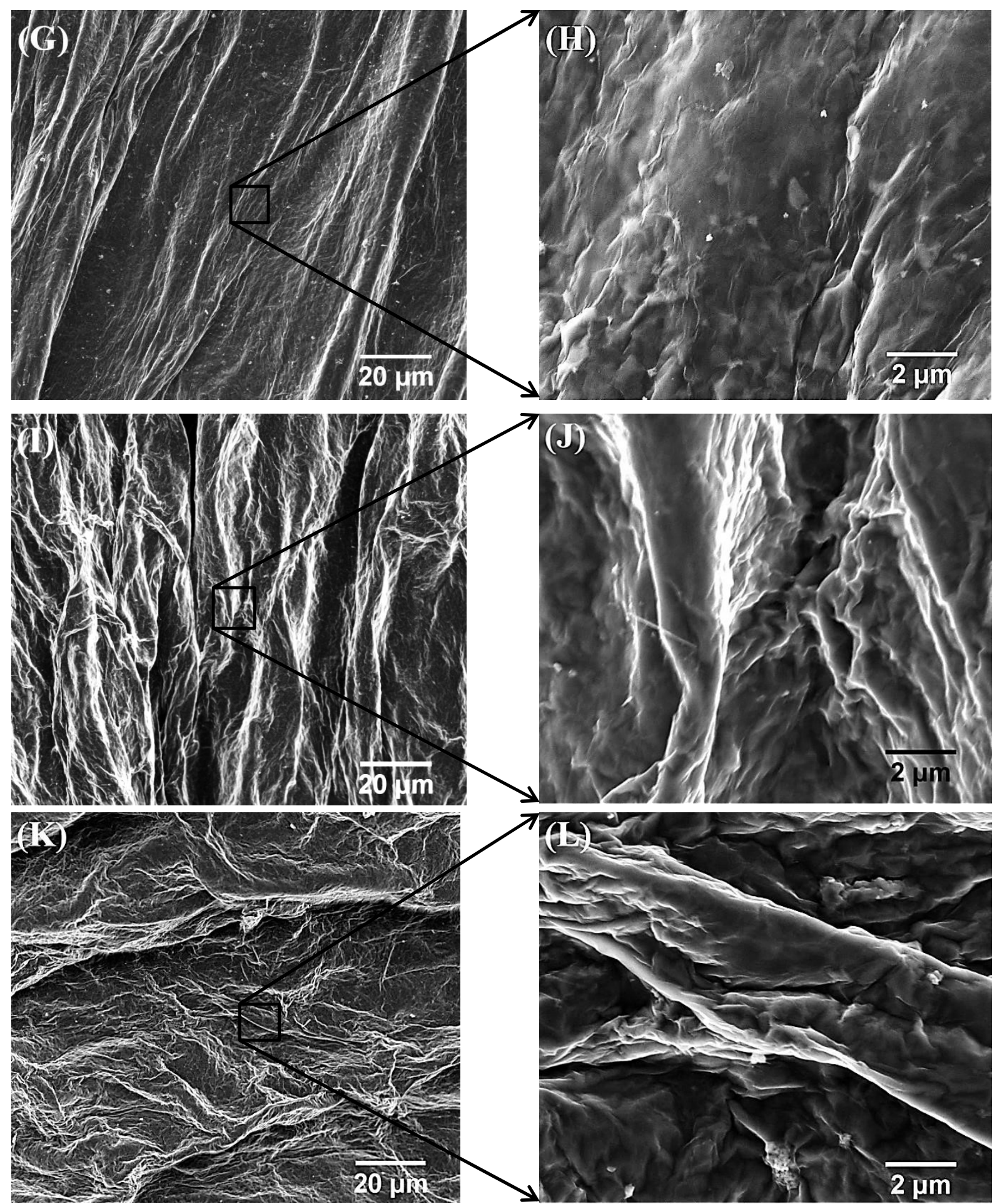

Figure 6. SEM images of the surface of (A, B) GO sheet, (C, D) 10k PEG, (E, F) N192h, (G, H) N192h-Ow, $(\mathrm{I}, \mathrm{J}) \mathrm{N} 72 \mathrm{~h}-\mathrm{Ow}$ and $(\mathrm{K}, \mathrm{L}) \mathrm{N} 24 \mathrm{~h}-0 \mathrm{w}$.

Figure 6 shows the SEM images of the fracture surfaces of the GO, 10k PEG, N192h, N192h-0w, N72h$\mathrm{Ow}$ and $\mathrm{N} 24 \mathrm{~h}-\mathrm{Ow}$, respectively. The images illustrate the changing of the fracture surface of nanohybrids compared to the $G O$ and PEG. Figure 6(A, B) presented a smooth surface with many small cracks on neat PEG, whereas Figure 6(C, D) clearly showed the flaky morphology surface of GO. The fracture surface of 
N192h was different to that of both PEG and GO, which showed shrinkage behaviour in Figure 6(E) and similar behaviour to that of the GO nanosheets in Figure 6(F) because the long mixing time and washing procedure removed most of the adsorption PEG from the GO surface. However, in Figure 6(G, H) the flaky fracture surface turned into densely packed sheets in the N192h-Ow surface because the polymer covered most of the GO nanosheet surface. Moreover, the adsorbed PEG was clearly observed on the GO nanosheets surface of N72h-Ow as shown in Figure 6(I, J). N24h-Ow showed much shrinkage on the surface due to the absorption of the PEG on the GO nanosheets. The PEG presented clearly on the GO nanosheets like wading. The SEM image clearly showed the N72h-Ow possessed more polymer compared to N192h in agreement with TGA results, with 38 wt\% PEG within the hybrid and a sharp DSC melting peak at $58.2^{\circ} \mathrm{C}$.
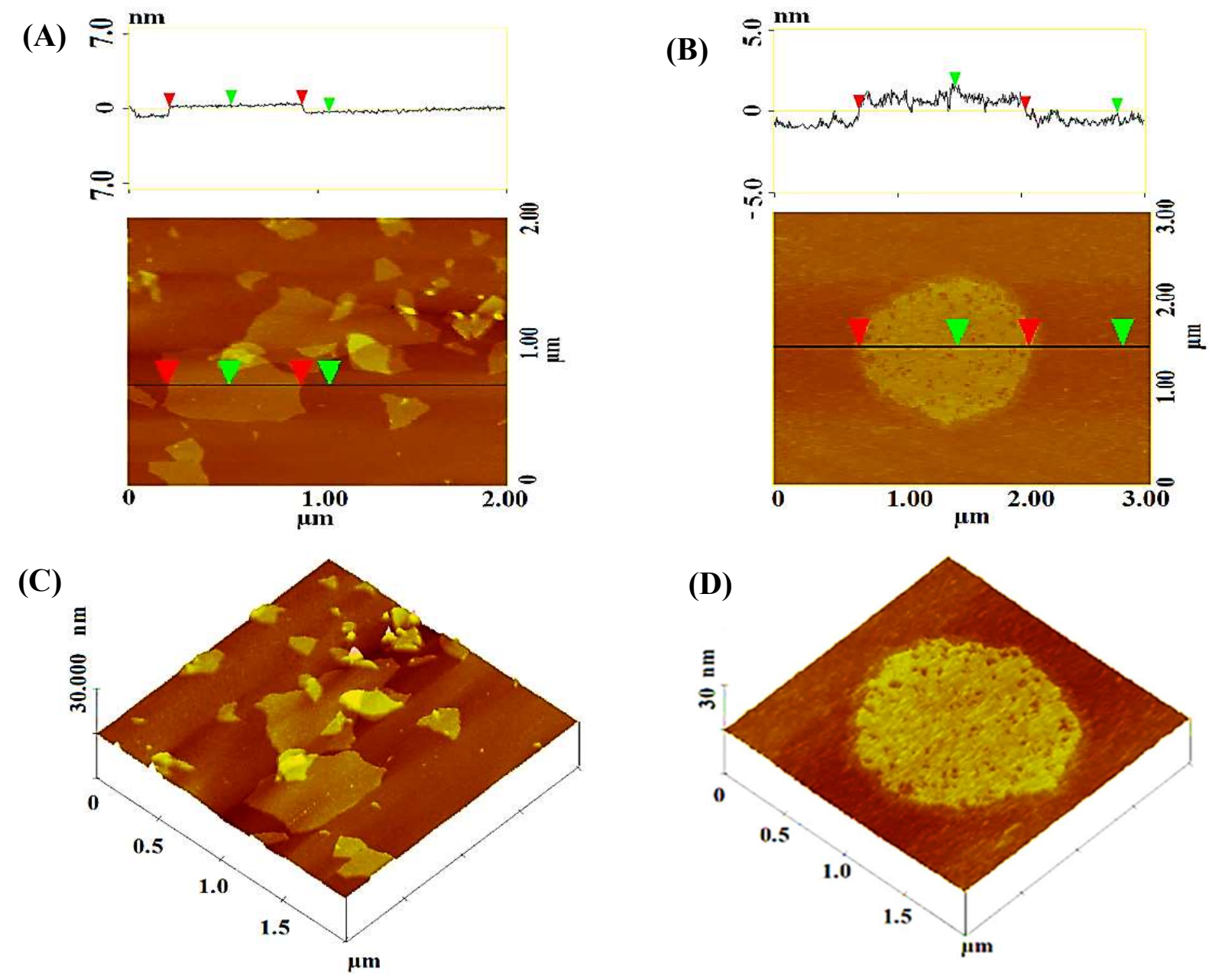

Figure 7. 2D and 3D AFM images of the (A, C) GO nanosheets and (B, D) N192-0w.

In Figure 7, the 2D and 3D AFM images characterised the morphology and thickness of GO and N192Ow. Figure $7(\mathrm{~A})$ shows the single sheets of $\mathrm{GO}$ with an average thickness of $0.77 \mathrm{~nm}$, which was close to the XRD results. The AFM image showed different shapes and sizes of GO nanosheets that were between 1840 and $150 \mathrm{~nm}$. In Figure 7(B), the surface roughness of N192-0w with a wrinkled structure 
is revealed. The thickness increases from $0.77 \mathrm{~nm}$ of GO to $1.58 \mathrm{~nm}$ of the $\mathrm{N} 192 \mathrm{~h}-0 \mathrm{w}$ due to adsorbed PEG onto the GO nanosheets, where the thickness of adsorbed PEG was $0.405 \mathrm{~nm}$ on each side of the GO nanosheet. The 3D AFM image of the GO nanosheets illustrated the roughness of the surface of the GO nanosheets, as shown in Figure $7(C)$, whereas the rough structure with wrinkle structure presented on the hybrid surface as a result of adsorbing the PEG on the GO nanosheets, as shown in Figure 7(D). These AFM images presented clear evidence and supported the results above, showing successful adsorption of PEG on the GO nanosheets.

\section{CONCLUSIONS}

A serial of PEG/GO hybrids was prepared using a solvent blending method with different parameters, including mixing time, polymer molecular weight, mixing ratio and washing procedure. The FTIR results confirmed the successful preparation of GO and the adsorption of PEG on GO nanosheets. The XRD results confirmed the intercalation of PEG between GO nanosheets for all samples but only presented the crystalline peak of the adsorbed PEG in the non-washed hybrids. Only the non-washed hybrids presented a melting transition of PEG in the samples. The thermal degradation temperature of PEG increased in all hybrids. The optical microscopic images confirmed the reduction of the crystalline size of the PEG. The SEM and AFM results presented strong evidence of PEG adsorption on the GO nanosheets.

The adsorption of PEG occurred relatively rapidly, whereas the long mixing time such as $192 \mathrm{~h}$ showed a negative effect on the polymer adsorption behaviour. The higher molecular weight significantly improved the polymer adsorption amount. Increasing the polymer mixing ratio also enhanced its adsorption amount. All the washed samples had mostly confined intercalated polymers between the GO nanosheets, and the application of a further washing procedure led to the removal of the adsorbed polymer from GO surface, causing a reduction in the polymer adsorption amount. Applying the optimal parameters simultaneously succeeded in achieving the highest polymer adsorption up to 38 wt.\%.

These findings may help understand the adsorption behaviour of the polymer onto GO nanosheets and the interfacial interaction between the polymer and GO and thus the macroscopic properties of the bulk polymer-GO nanocomposites. They may also serve as guidance in future design and manufacture of polymer-GO nanocomposites for different applications.

\section{ACKNOWLEDGEMENTS}

E. Al-Bermany would like to thank The University of Babylon, the Iraqi Ministry of Higher Education and Scientific Researches for funding his PhD scholarship. 


\section{REFERENCES}

1. Mohammad, M., Winey, K. I. Macromolecules, 2006, 39, 5194-5205.

2. Krishnamoorti, R., Vaia, R. A. Journal of Polymer Science: Part B: Polymer Physics, 2007, 45, 3252-3256.

3. Abdelamir, A. I., Al-Bermany, E., Hashim, F. S. Journal of Physics: Conference Series, 2019, 1294, 022029, 1-13.

4. Roth, C. B., McNerny, K. L., Jager, W. F., Torkelson, J. M. Nature Materials, 2007, 6, 257-258.

5. Al-Bermany, A.-K. J., Al-Bermany, E., Kadem, B. Y. European Journal of Scientific Research, 2011, 61, 203-209.

6. Zou, G., Luo, H., Sun, B., Jain, M., Peng, H. Nanotechnology, 2011, 2011, 1-2.

7. Jawad, E., Khudhair, S. H., Ali, H. N. European Journal of Scientific Research, 2011, 60, 63-70.

8. Ranjbartoreh, A. R., Wang, B., Shen, X., Wang, G. Journal of Applied Physics, 2011, 109, 014306, 1-6.

9. Stankovich, S., Piner, R. D., Nguyen, S. T., Ruoff, R. S. Carbon, 2006, 44, 3342-3347.

10. Al-Bermany, E., Qais, D., Al-Rubaye, S. Journal of Physics: Conference Series, 2019, 1234, 012011, 1-12.

11. Abdelamir, A. I., Al-Bermany, E., Hashim, F. S. AIP Conference Proceedings, 2020, 2213, 020110, $1-13$.

12. Hong, B. J., Compton, O. C., Zhi An, I. E., Nguyen, S. T. ACS Nano, 2012, 6, 63-73.

13. Wan, C., Chen, B. Journal of Materials Chemistry, 2012, 22, 3637-3646.

14. Abdul, M., Al-bermany, E. Journal of Green Engineering, 2020, 10, 3465-3483.

15. Rittigstein, P., Priestley, R. D., Broadbelt, L. J., Torkelson, J. M. Nature materials, 2007, 6, 278282.

16. Al-nesrawya, S. H., Mohseenb, M. J., Al-Bermany, E. IOP Conference Series: Materials Science and Engineering, 2020, 871, 012060, 1-11.

17. Chen, B., Evans, J. R. G. Macromolecules, 2006, 39, 1790-1796.

18. Barroso-Bujans, F., Fernandez-Alonso, F., Cerveny, S., Parker, S., Alegria, A., Colmenero, J. Soft Matter, 2011, 7, 7173-7176.

19. Barroso-Bujans, F., Fernandez-Alonso, F., Pomposo, J. A., Enciso, E., Fierro, J. L. G., Colmenero, J. Carbon, 2012, 50, 5232-5241.

20. Barroso-Bujans, F., Fernandez-Alonso, F., Pomposo, J. A., Cerveny, S., Alegría, A., Colmenero, J. ACS Macro Letters, 2012, 1, 550-554.

21. Barroso-Bujans, F., Fernandez-Alonso, F., Cerveny, S., Arrese-Igor, S., Alegría, A., Colmenero, J. Macromolecules, 2012, 45, 3137-3144.

22. Vaia, R. A., Sauer, B., Tse, O., Giannelis, E. P. Journal of Polymer Science: Part B: Polymer Physics, 1997, 35, 59-67.

23. Miwa, Y., Drews, A. R., Schlick, S. Macromolecules, 2008, 41, 4701-4708.

24. Su, C. C., Shen, Y. H. Journal of Colloid and Interface Science, 2009, 332, 11-15.

25. Koksal, E., Ramachandran, R., Somasundaran, P., Maltesh, C. Powder Technology, 1990, 62, 253-259.

26. Mathur, S., Moudgil, B. M. Journal of Colloid and Interface Science, 1997, 196, 92-98.

27. Yuang, P. C., Shen, Y. H. Journal of Colloid and Interface Science, 2005, 285, 443-447.

28. Chen, B., Evans, J. R. G. Journal of Physical Chemistry B, 2004, 108, 14986-14990.

29. Pei, X., Zhu, Z., Gan, Z., Chen, J., Zhang, X., Cheng, X., Wan, Q., Wang, J. Scientific Reports, 2020, 10, 2717, 1-15.

30. Sun, X., Liu, Z., Welsher, K., Robinson, J. T., Goodwin, A., Zaric, S., Dai, H. Nano Research, 2008, 1, 203-212.

31. Li, Y., Li, Y., Huang, X., Zheng, H., Lu, G., Xi, Z., Wang, G. Composites Science and Technology, 2020, 195, 10819, 1-10.

32. Chen, B., Evans, J. R., Holding, S. Journal of Applied Polymer Science, 2004, 94, 548-552.

33. Hummers, W. S., Offeman, R. E. Journal of the American Chemical Society, 1958, 80, 1339. 
34. Wang, G., Shen, X., Wang, B., Yao, J., Park, J. Carbon, 2009, 47, 1359-1364.

35. Ganesh, B. M., Isloor, A. M., Ismail, A. F. Desalination, 2013, 313, 199-207.

36. Wang, C., Feng, L., Yang, H., Xin, G., Li, W., Zheng, J., Tian, W., Li, X. Physical Chemistry Chemical Physics, 2012, 14, 13233-13238.

37. Gao, S., Zhong, J., Xue, G., Wang, B. Journal of Membrane Science, 2014, 470, 316-322.

38. Yang, Y., Zhou, C.-H., Xu, S., Zhang, J., Wu, S.-J., Hu, H., Chen, B.-L., Tai, Q.-D., Sun, Z.-H., Liu, W., Zhao, X.-Z. Nanotechnology, 2009, 20, 105204, 1-9.

39. Potts, J. R., Dreyer, D. R., Bielawski, C. W., Ruoff, R. S. Polymer, 2011, 52, 5-25.

40. Pulst, M., Samiullah, M. H., Baumeister, U., Prehm, M., Balko, J., Thurn-Albrecht, T., Busse, K., Golitsyn, Y., Reichert, D., Kressler, J. Macromolecules, 2016, 49, 6609-6620.

41. Winey, K. I., Vaia, R. A. MRS Bulletin, 2007, 32, 314-358.

42. Li, Y., Louarn, G., Aubert, P. H., Alain-Rizzo, V., Galmiche, L., Audebert, P., Miomandre, F. Carbon, 2016, 105, 510-520.

43. Al-Bermany, E. Doctoral dissertation, University of Sheffield, 2017.

44. Vaia, R. A., Liu, W. Journal of Polymer Science, Part B: Polymer Physics, 2002, 40, 1590-1600.

45. Godovsky, Y. K., Slonimsky, G. L., Garbar, N. M. Journal of Polymer Science Part C: Polymer Symposia, 1972, 38, 1-21.

46. Nelson, A., Cosgrove, T. Langmuir, 2004, 20, 10382-10388.

47. Wan, C., Chen, B. Biomedical Materials, 2011, 6, 055010, 1-8.

48. Jagtap, S. B., Kushwaha, R. K., Ratna, D. RSC Advances, 2015, 5, 30555-30563.

49. Cai, D., Yusoh, K., Song, M. Nanotechnology, 2009, 20, 085712, 1-5.

50. Pavlidou, S., Papaspyrides, C. D. Progress in Polymer Science, 2008, 33, 1119-1198.

51. Tong, Y., Lin, Y., Wang, S., Song, M. Polymer, 2015, 73, 52-61.

52. Luo, Z., Cong, C., Zhang, J., Xiong, Q., Yu, T. Carbon, 2012, 50, 4252-4258. 